\title{
Intermédialités
}

Histoire et théorie des arts, des lettres et des techniques

Intermediality

History and Theory of the Arts, Literature and Technologies

\section{Entre textes urbains et critique cinématographique : Kracauer scénariste de la ville}

\section{Nia Perivolaropoulou}

Numéro 14, automne 2009

bâtir

build

URI : https://id.erudit.org/iderudit/044407ar

DOI : https://doi.org/10.7202/044407ar

Aller au sommaire du numéro

Éditeur(s)

Revue Intermédialités (Presses de l’Université de Montréal)

ISSN

1705-8546 (imprimé)

1920-3136 (numérique)

Découvrir la revue

Citer cet article

Perivolaropoulou, N. (2009). Entre textes urbains et critique

cinématographique : Kracauer scénariste de la ville. Intermédialités /

Intermediality, (14), 19-35. https://doi.org/10.7202/044407ar
Résumé de l'article

Les rapports entre la ville et le cinéma chez Siegfried Kracauer ont été abordés surtout à partir de ses textes sur la ville et de ses essais sur les médias photographiques des années 1920 et 1930. Le présent article se propose d'éclairer plus avant les liens entre textes urbains, critiques de film et élaboration théorique, en se penchant sur de courts textes, négligés par la recherche, dans lesquels Kracauer esquisse des projets de scénarios. La contextualisation de ces textes destinés à la production cinématographique et leur mise en perspective historique mettent à jour les déplacements et les transformations, dans un mouvement à la fois de continuité et de discontinuité, des concepts kracaueriens établis dès les années 1920. Parallèlement à la mise en place de la notion centrale de perception estrangée, on voit se dessiner la centralité du médium film pour la pensée de Kracauer. 


\title{
Entre textes urbains et critique cinématographique: Kracauer scénariste de la ville
}

\author{
Nia Perivolaropoulou
}

G eorg Simmel, Siegfried Kracauer et Walter Benjamin sont trois penseurs de la grande ville et de la modernité qui ont marqué leur temps et continuent à nourrir les discussions sur le devenir des métropoles et notre condition actuelle. Architecte de formation, Kracauer ne développe cependant pas sa pensée de la ville dans le cadre de son métier, qu'il n’a exercé que de façon éphémère et pour des raisons alimentaires. Tout au contraire. C'est à partir du moment où il a pu le quitter et travailler régulièrement pour le feuilleton du grand journal libéral de la République de Weimar, la Frankfurter Zeitung, que sa pensée va se déployer. Le feuilleton de la FZ représente un forum de réflexion sur la société allemande d'après-guerre, l'avènement de la modernité et la conscience de crise qui l'accompagne; mais il devient aussi le lieu d'expérimentation de nouvelles formes d'écriture, à même de rendre compte des phénomènes nouveaux.

Pour Kracauer, comme pour Walter Benjamin, la grande ville, qui bouleverse les modes de vie traditionnels, et l'apparition de la photographie et du cinéma, qui constituent une rupture avec l'art, participent du processus de la modernité. Très tôt en effet, la grande ville, les manifestations de la culture de masse et les «médias photographiques» cristallisent la réflexion de Kracauer sur les mutations des cadres de la sensibilité, des modes de la perception et des possibilités de l'expérience du sujet dans les conditions de la modernité.

Tout au long des années 1920 et jusqu'à son exil en mars 1933, après la prise du pouvoir par Hitler, Kracauer ne cesse d'observer les transformations de la grande ville et de ses représentations, les nouveaux modes de communication, les nouvelles pratiques de la vie quotidienne, les distractions, enfin, des nouvelles classes moyennes urbaines. Tout en renouvelant profondément les sujets du feuilleton, en élargissant les domaines traditionnels de la littérature et des arts 
pour y inclure, par exemple, des genres réputés triviaux, tels le roman policier, et surtout en développant la critique de cinéma.

Dans ses articles rédigés au jour le jour, Kracauer se sert de formes d'écriture qui vont du grand essai philosophique aux miniatures littéraires, en passant par la critique littéraire ou cinématographique, les textes de flânerie, les portraits de ville. Au fil du temps, le cinéma, les phénomènes nouveaux, singuliers, de la vie urbaine et les transformations continues et quasi imperceptibles de la grande ville occupent une place de plus en plus centrale dans sa production.

La grande crise économique de 1929 ainsi que l'évolution politique en Allemagne entraînent des transformations profondes au sein du journal. Kracauer est amené dans un premier temps à quitter Francfort pour intégrer la rédaction du feuilleton à Berlin, et bientôt il subit, ainsi que d'autres collaborateurs, des pressions croissantes et des diminutions successives de salaire. Aussi, en décembre 1931, la direction du journal lui conseille-t-elle de se préoccuper de trouver une activité rémunérée complémentaire. Heinrich Simon, l'éditeur du journal, essaie alors de persuader Kracauer de collaborer avec la Ufa, et va jusqu'à envisager son détachement pour une année auprès de la société de production cinématographique allemande. Si les motivations de Simon ne sont pas évidentes, le gain pour la Ufa l'est ${ }^{1}$. Car Kracauer, en même temps qu'il traquait dans ses critiques les idéologies véhiculées en catimini dans les films allemands en premier lieu, et les productions de la Ufa en particulier, avait multiplié les articles sur l'industrie du cinéma et ses rapports avec la politique, comme sur les pratiques de la censure.

Si l'éventualité d'une telle collaboration tourne court rapidement, Kracauer va faire sienne la proposition d'écrire pour la production cinématographique 2 . Entre 1932 et 1938, il conçoit quatre projets filmiques, fort différents les uns des

1. Voir sur ce sujet Inka Mülder-Bach, «Nachbemerkungen und editorische Notiz», dans Siegfried Kracauer, Werke, vol. 6.3: Kleine Schriften zum Film 1932-1961, édité par Inka Mülder-Bach, Francfort, Suhrkamp, 2004, p. 575-577. Dans une lettre adressée à Bernhard Guttmann, alors directeur de la FZ à Berlin, datée du 1.1.1932, Kracauer écrit: «Pas de doute qu'elle [la Ufa] dépenserait principalement son argent pour me réduire au silence pendant ce laps de temps; car depuis toujours j'ai combattu les tendances qui dominent en son sein et je ne pourrais évidemment pas, pendant ce temps-là, écrire des critiques de film», ibid., p. 576.

2. Sa décision a peut-être été motivée par sa volonté de contrecarrer les projets de collaboration avec la Ufa et par la perspective d'améliorer ainsi, éventuellement, sa situation financière. À cette occasion, Kracauer allie encore une fois contrainte et occasion d'expérimentation. 
autres: le premier consiste en des propositions de courts métrages destinés au programme des cinémas avant la projection du film principal; le deuxième et le troisième sont deux synopsis, l'un pour une adaptation cinématographique du roman d'Alphonse Daudet Tartarin sur les Alpes, l'autre pour un moyen métrage intitulé Sonntag/Dimanche; le dernier enfin, le plus développé, est un film treatment en vue d'une adaptation au cinéma de son ouvrage Jacques Offenbach ou le secret du Second Empire 3 . Deux de ces projets, les propositions de courts métrages et Dimanche, s'inscrivent directement dans la lignée des textes - essais, critiques de films ou miniatures urbaines - qui relèvent des affinités entre grande ville et cinéma. Celles-ci, multiples, reposent, entre autres, sur leurs temporalités respectives et sur tout ce qui rapproche le citadin et le spectateur en tant que sujets d'une «perception dans la distraction ». Et si la ville est par excellence un espace cinématographique, les salles de cinéma avec leurs enseignes lumineuses participent à la configuration du paysage urbain.

Minuscules projets qui font le pont entre l'Allemagne et l'exil, les propositions de courts métrages et Dimanche se situent au croisement des essais critiques et des textes de ville dans lesquels Kracauer expérimente les formes d'écriture aptes à représenter la métropole moderne 4 . Par là même, ils attirent l'attention sur des aspects de ses critiques de films le plus souvent relégués au second plan derrière la critique idéologique. Intégrant les dimensions sociologique et esthétique, ils leur répondent en écho, tout en tissant des liens avec les autres textes de Kracauer et en anticipant, déjà, certains développements ultérieurs qu'on retrouve dans Theory of Film. Ténus passages entre essais, critiques de films et textes littéraires,

3. Les trois premiers, «Exposé [zu einer Kurzfilm-Serie] », «Ideen-Entwurf zu einer "grossen Filmkomödie" nach dem berühmten Roman: "Tartarin sur les Alpes" von Alphonse Daudet», et «Dimanche [Sonntag] », sont publiés dans Kracauer, 2004, vol. 6.3, respectivement p. 515 et sq., p. 518-522 et p. 523-626. De ce dernier texte, écrit dans les premiers mois de l'exil parisien, il existe deux versions, l'une en allemand, l'autre en français. Le film treatment est publié dans Siegfried Kracauer, Werke, vol. 8: Jacques Offenbach und das Paris seiner Zeit, édité par Ingrid Belke, Francfort, Suhrkamp, 2005, p. 485-502. Sur ce dernier projet, voir Nia Perivolaropoulou, «Les Jacques Offenbach de Siegfried Kracauer. Biographie, histoire et cinéma », dans Philippe Despoix et Peter Schötller (dir.), Siegfried Kracauer, penseur de l'histoire, Paris et Québec, Éditions de la Maison des sciences de l'homme et Presses de l'Université Laval, 2006, p. 165-186.

4. Voir en particulier Philippe Despoix, «Siegfried Kracauer, chroniqueur de l'époque de Weimar», dans Siegfried Kracauer, Le voyage et la danse. Figures de ville et vues de films, textes choisis et présentés par Philippe Despoix, trad. Sabine Cornille, Paris et Québec, Éditions de la Maison des sciences de l'homme et Presses de l'Université Laval, 2008, p. 5-20. 
ils condensent en eux l'expérience de Kracauer flâneur, critique et spectateur de cinéma.

Je me propose de présenter ces deux projets en explicitant les liens qu'ils peuvent entretenir avec les textes de ville et les critiques de films, afin d'apporter un éclairage supplémentaire sur l'intrication des différentes approches de la ville chez Kracauer; mais aussi, en les mettant en perspective du point de vue de son grand ouvrage, publié en 1960 aux États-Unis, Theory of Film. The Redemption of Physical Reality ${ }^{5}$, de tenter d'esquisser les lignes de développement de sa pensée sur les affinités entre la ville et le cinéma en suivant les déplacements de la notion de visibilité et les transformations de sa conception d'une "perception dans la distraction ", jusqu'à l'abandon de cette notion forgée dans les années de Weimar au profit de celle de la perception estrangée.

\section{L'ÉTRANGETÉ DES VILLES}

Le projet de la série de courts métrages, qui date de février 1932, comporte trois parties, dont la première expose l'argument d'un seul film, la deuxième présente de façon lapidaire les sujets d'une série de quatre films, et la troisième suggère la voie pour produire, à partir d'un même principe, d'autres séries. Considérés ensemble, ces sujets disparates dessinent une topographie filmique de la ville, mais une topographie implicite, qui ne se dévoile qu'en les mettant en relation avec d'autres textes de Kracauer, qu'à leur tour ils éclairent.

Les langues et les villes: «Monsieur X visite Paris ${ }^{6} »-$ l'intitulé du premier court métrage pourrait aussi bien être le titre d'une leçon de la méthode Assimil. Le film se présente comme «une version filmique à la fois amusante et instructive des modes d'emploi contenus ou non dans le Bädecker ${ }^{7}$ » Il devait montrer les petites mésaventures d'un touriste allemand dès son arrivée à Paris et pourrait se terminer, écrit Kracauer, avec le récit des impressions de voyage du personnage, une fois rentré à la maison, qu'accompagneraient des images de son voyage, mais, précise-t-il, déformées. Si dans ce premier court métrage Monsieur X est allemand et la ville est Paris, Kracauer suggère que les deux

5. Siegfried Kracauer, Theory of Film. The Redemption of Physical Reality, New York, Oxford University Press, 1960. La traduction française vient de paraître: Siegfried Kracauer, Théorie du film. La rédemption de la réalité matérielle, trad. Daniel Blanchard et Claude Orsoni, édité et présenté par Philippe Despoix et Nia Perivolaropoulou, Paris, Flammarion, coll. «Bibliothèque des savoirs», 2010.

6. «Monsieur X fährt nach Paris?», Kracauer, 2004, vol. 6.3, p. 515.

7. Ibid. 
éléments sont interchangeables. Le personnage, au cas où le film rencontrerait le succès, pourrait se styliser en un «type» et devenir le héros d'une série. La façon la plus simple et la plus économique pour produire une telle série étant de faire des versions différentes du même film, c'est-à-dire de changer la nationalité de Monsieur X et par conséquent la langue. On comprend alors pourquoi Kracauer, dès la première phrase, attire l'attention sur la bande-son. En effet, le film devait être «très joli aussi sur le plan sonore ${ }^{8}$ ».

Cette dernière remarque rappelle tout l'intérêt que porte Kracauer, dès les débuts du film sonore, à l'utilisation filmique du son. Outre ses réflexions plus générales et les comparaisons entre les productions allemandes, françaises ou américaines qu’on trouve disséminées dans différents textes sur le cinéma, il revient sur ce problème à maintes reprises dans ses critiques des premiers films sonores. Quasi contemporain de son projet de courts métrages, le film de Julien Duvivier, Hallo! Hallo! Hier spricht Berlin, raconte une histoire d'amour, par voix interposées, entre un téléphoniste berlinois et sa collègue française. Le film n’est qu’une série de quiproquos qui se veulent amusants, écrit Kracauer dans la brève critique qu'il lui a consacrée9. Duvivier ne possédant ni le talent ni la légèreté d'un René Clair, les types dans son film sont grossiers et le rire parfois gras. Mais il est basé sur «une heureuse idée de film sonore, la confrontation des langues ${ }^{10}$ ».

Une scène toutefois trouve grâce, sur le plan visuel, à ses yeux, celle du tour obligatoire à travers Paris, dans laquelle les touristes «font» en un clin d'œil l'Opéra, la Madeleine et la tour Eiffel. La mode des voyages touristiques avait été, jusqu'au milieu des années 1920, interprétée par Kracauer comme une «passion spatio-temporelle», expression du processus de désenchantement propre à la modernitée ${ }^{11}$. Au fil du temps, alors même que se modifiait dans l'ensemble son attitude vis-à-vis des phénomènes de la culture de masse et avant tout du cinéma,

\section{Ibid.}

9. Le titre original est Allo Berlin? Ici Paris! (France, 1931-1932). La critique de ce film, intitulée «Paris-Berlin », est intégrée dans le compte rendu groupé «Einige Filme» (22.3.1932), dans Kracauer, 2004, p. 37-41.

10. Ibid., p. 40.

11. Rappelons que dans un premier temps l'avènement de la modernité est considéré par Kracauer comme un processus fondamentalement négatif, comme une perte du sens transcendantal. Dans cette perspective, il voit dans les rythmes des danses à la mode ou du jazz et dans le goût pour les voyages l'expression du culte du mouvement pour lui-même et d'un temps vide, à la fois «excès de nature théologique» et "phénomènes provisoires à caractères profanes». Voir «Le voyage et la danse» (15.3.1925), dans Kracauer, 2008, p. 21-30. 
son jugement sur les voyages s'est également nuancé. Si cet aspect de critique des voyages organisés est présent dans son article sur le film de Duvivier, Kracauer y pointe incidemment un problème bien différent: la non-perception de l'espace environnant. Les touristes du film de Duvivier qui se pressent devant les monuments ne les voient pas vraiment. De même, le récit oral des impressions de voyage de Monsieur X devait s'accompagner des images déformées qui lui correspondent, parce que littéralement celui-ci ne voyait pas la réalité qui l'entourait; alors que toute la partie du court métrage dépeignant ses aventures à Paris aurait montré la ville en même temps que l'incapacité du personnage de Monsieur X à affronter ce qui lui est étranger. La non-perception de la réalité des villes, que les clichés, les représentations stéréotypées en tous genres, langagières ou imagées, ainsi que les comportements devenus naturels peuvent littéralement recouvrir, va peut-être en s'aggravant, constate Kracauer. Nombre de représentations filmiques y contribuent, déplore-t-il, alors que le cinéma est, selon lui, le médium adéquat pour représenter la ville, pour lui rendre sa visibilité ${ }^{12}$.

Des années plus tard, dans Théorie du film, la confrontation des langues et l'expérience de l'étranger vont occuper une place centrale dans ses réflexions. Pour rendre perceptible l'effet que peut avoir un film où l'on parle plusieurs langues, Kracauer cite longuement un passage des Praeterita de John Ruskin:

«There is something peculiarly delightful in passing through the streets of a foreign city without understanding a word that anybody says! One's ear for all sound of voices then becomes entirely impartial; one is not diverted by the meaning of syllables from recognizing the absolute guttural, liquid, or honeyed quality of them: while the gesture of the body and the expression of the face have the same value for you that they have in a pantomime; every scene becomes a melodious opera to you, or a picturesquely inarticulate Punch". ${ }^{13}$ ”

Le découplage de la sonorité des mots de leur signification offre, ainsi que Kracauer le montre dans Théorie du film, la possibilité d'une utilisation proprement cinématographique des dialogues et du langage en général. Cette

12. On pourrait multiplier les références. On mentionnera ici sa critique du film de Carmine Gallone, une coproduction germano-britannique intitulée Die singende Stadt (The City of Song, 1930), exemple à la fois d'un emploi non cinématographique du son, d'une représentation «carte postale» des villes italiennes et d'une utilisation de la musique, ici les airs d'opéra, comme procédé de recouvrement. Voir «Über den musikalischen Tonfilm» (2.11.1930), dans Siegfried Kracauer, vol. 6.2 : Kleine Schriften zum Film 1928-1931, édité par Inka Mülder-Bach, Francfort, Suhrkamp, 2004, p. 410-412.

13. John Ruskin, Praeterita, Londres, Hart-Davis, 1949, p. 106, cité d'après Theory of Film, p. 109-110. 
considération formelle implique ipso facto une dimension cognitive, un tel fonctionnement de la langue ouvrant sur une perception estrangée de la réalité. Dans l'expérience esthétique décrite par Ruskin, la suspension du sens des mots, en rendant à la langue sa qualité sonore, est susceptible d'induire une perception différente de la ville, une perception de son étrangeté.

Kracauer élargira encore dans l'ouvrage son propos. En évoquant les films tournés après la Deuxième Guerre mondiale où l'on parle plusieurs langues, il montre que ce même découplage constitue une condition de la perception de l'autre, de l'étranger. Perception qui passe par le rapprochement physique, instinctif, corporel, sensuel et émotionnel. Le premier épisode de Païsa (1946) de Rossellini, qui met en scène la rencontre d'une jeune Italienne du Sud et d'un GI, est alors convoqué pour l'illustrer.

Les sons de la ville: parmi la production des premières années du cinéma sonore, deux films se distinguent pour leur utilisation du son, de la parole et de la musique: Hallelujah (1929) de King Vidor et Sous les toits de Paris (1930) de René Clair sur lesquels Kracauer revient à plusieurs reprises dès leur sortie ${ }^{14}$. Dans sa critique du film de Clair, il rappelle que celui-ci raconte une histoire inspirée d'une rengaine homonyme de Raoul Moretti et René Nazelles. Elle se déroule dans un quartier populaire de Paris, parmi des marginaux qui vivent parfois à la frontière de la légalité; ceux-ci fusionnent presque avec les rues dans lesquelles ils traînent, les bals musettes qu'ils fréquentent et les chambres sous les toits qu'ils habitent. Pour Kracauer, après avoir montré dans Entr'Acte (1924) l'existence ordinaire selon la stricte logique du rêve, René Clair fait dans ce film quelques concessions aux formes établies et n'évite pas toujours la sentimentalité. Mais Clair réussit en même temps deux choses importantes: d'abord une utilisation véritablement filmique du son, et en cela il devrait constituer un modèle pour les cinéastes allemands; ensuite il donne à voir la ville: « $\mathrm{Ce}$ n'est pas l'action qui se joue à Paris, mais Paris qui joue cette action. Au lieu d'être elle-même le centre, elle est simple rayonnement à partir d'un autre centre, difficilement saisissable. Elle se produit comme par hasard sur le pavé des rues,

14. Voir la critique de Hallelujah dans Kracauer, 2004, vol. 6.2, p. $401-405$ (ainsi que les mentions dans Theory of Film). Kracauer consacre une critique au film de René Clair à sa sortie en Allemagne, voir «Neue Tonfilme. Einige grundsätzliche Bemerkungen» (17.8.1930), ibid., p. 392-397 (traduction française: «Sous les toits de Paris», dans Kracauer, 2008, p. 111-114). Mais il y revient à plusieurs reprises, quand il aborde les problèmes du film sonore). Voir «Über den Tonfilm» (24.12.1930), ibid., p. 434-436; «Zur Lage des Tonfilms» (février 1931), ibid., p. 462-466; «Internationaler Tonfilm?» (mars 1931), ibid., p. 475-479; et «Die verflossene Tonfilmsaison» (août 1931), ibid., p. 530-535. 
sous les rangées d'ampoules électriques des cabarets et dans de sombres cages d'escaliers ${ }^{15}$.» Dans le film de Clair, la musique et les images sont inextricablement liées, mais selon un mode qui n'est pas celui de la détermination réciproque obéissant à une logique exclusivement narrative.

Quelques mois après avoir écrit sa critique du film de René Clair, Kracauer évoque, dans un texte où, en méditant sur le destin des tubes, il aborde le problème de leurs effets chez les auditeurs, la musique d'un orgue de barbarie qui parvient jusqu'à lui, malgré le bruit des machines à écrire et des pas qui résonnent dans les couloirs, à travers les fenêtres fermées de son bureau à Berlin. La musique déclenche en lui, raconte-t-il, le souvenir d'une après-midi à Paris, dans une petite rue derrière le Palais-Royal. Une femme, accompagnée de deux musiciens, chantait un tube, repris par le public constitué au hasard dans la rue. La chanson était à la gloire des aviateurs Dieudonné Costes et Maurice Bellonte, qui venaient de réussir le premier vol Paris-New York sans escale. En chantant, le rassemblement disparate, regroupant artisans, petites bourgeoises ou jeunes filous, se transformait en une quasi-communauté. La scène avait alors semblé familière à Kracauer, qui pourtant ne se souvenait pas d'avoir jamais participé à semblable rassemblement. Soudainement, alors que les gens reprenaient de plus belle le tube du moment, cela lui est revenu: c'était dans le film de René Clair, Sous les toits de Paris, qu'il avait vu à Berlin peu de temps avant son voyage à Paris. Maintenant, après son retour, la même mélodie essayait de remonter le long des murs de l'immeuble des bureaux berlinois de la FZ, en vain. Elle était étrangère. Partie de Paris, elle avait voyagé avec le cinéma, «là où elle était encore à moitié chez elle ${ }^{16} »$. Sortie des salles de cinéma, elle avait trouvé refuge pour un moment dans les cafés et les salons, avant de retourner dans la rue. Mais dans ces rues personne ne la reconnaissait ni ne la chantonnait. Les tubes sont des êtres indépendants et voyageurs; ils se détachent de leur contexte d'origine, hantent des lieux lointains et des gens dans les quatre coins du monde. Puis ils disparaissent, et parfois ils reviennent. Leurs significations sont aussi labiles qu'ils sont vagabonds. Ils peuvent bien alors, tels les humains, se retrouver en exil ${ }^{17}$.

Considérés ensemble, la critique du film de René Clair et le texte sur les «tubes en exil» établissent une homologie entre la relation qu'entretiennent

15. «Sous les toits de Paris», Kracauer, 2008, p. 112.

16. «Schlager im Exil»(24.2.1931), dans Siegfried Kracauer, Schriften, vol. 5.2: Aufsätze 1927-1931, édité par Inka Mülder-Bach, Francfort, Suhrkamp, 1990, p. 287.

17. Sur les tubes, voir Peter Szendy, Tubes. La philosophie dans le juke-box, Paris, Les Éditions de Minuit, coll. «Paradoxe», 2008. 
la bande-son du film et l'espace acoustique de la ville et celle qui existe entre la ville représentée à l'écran et la ville réelle. Si dans le film de René Clair la musique et les images sont dans un rapport indéterminé, elles sont cependant liées. La musique de ce tube est constitutive de l'espace filmique urbain. Plus, elle contribue, avec les espaces représentés, les dancings, les sombres cages d'escaliers, etc., à dessiner ce centre fuyant de la ville, qu'on pourrait, dans une première approximation, décrire comme la co-appartenance des lieux et de leurs habitants. Dans le film et dans Paris. Tout comme les souvenirs des villes et les souvenirs des films s'entremêlent inextricablement chez Kracauer.

\section{ESPACE URBAIN, ESPACE SOCIAL}

Pour présenter en quelques lignes l'ensemble des quatre sujets qui constituent la deuxième partie de son projet de courts métrages, Kracauer recourt aux mêmes expressions que dans l'introduction de son étude sur les employés ${ }^{18}$, en même temps qu'il leur assigne une fonction similaire: la représentation sociologiquement pertinente du milieu de vie immédiat. Ces courts métrages devaient explorer «l'exotisme de la quotidienneté» et s'opposer ainsi aux documentaires et films de fiction empreints d'exotisme ${ }^{19}$. Quantité de textes de Kracauer de cette période sont de brefs, voire de minuscules récits de ses expéditions dans la quotidienneté urbaine, dont la forme cherche à en donner une représentation. Ces courts métrages sonores apparaissent alors d'emblée comme conçus pour faire pendant aux textes de ville.

1. «Sur les toits de Berlin» se propose de filmer les belles formes des toits berlinois ainsi que quelques appartements sous les toits. Le film projeté se situe aux antipodes de «Sous les toits de Paris ${ }^{20}$ ». Linversion de «sous» en «sur» a valeur d'indice, même si le court métrage envisagé n’est en rien comparable avec le film de René Clair. Dans ce dernier, «l'œil peut sans obstacle descendre du haut de la forêt des cheminées sur les toits jusqu'à la foule populaire en train

18. Siegfried Kracauer, Les employés. Aperçus de l'Allemagne nouvelle [1929], trad. Claude Orsoni, édité et présenté par Nia Perivolaropoulou, Paris, Éditions de la Maison des sciences de l'homme-Avinus, 2004.

19. Peu de temps après, la notion d'«Exotik des Alltags» est reprise et systématiquement développée en articulation avec la question du reportage ; voir «Reisen, nüchtern » (10.7.1932), dans Siegfried Kracauer, Schriften, vol. 5.3: Aufsätze 1932-1965, édité par Inka Mülder-Bach, Francfort, Suhrkamp, 1990, p. 87-90.

20. «Über den Dächern von Berlin », Kracauer, 2004, vol. 6.3, p. 516. 
de chanter ${ }^{21}$ ». À l'inverse, dans sa première proposition de reportage filmique urbain, Kracauer délaisse résolument la ville en bas, et avec elle les Berlinois, pour s'élever pendant un moment au-dessus des conditions réelles de vie et se mouvoir entre les toits de Berlin. Leurs formes semblent attirer son regard, comme les ornements en fer forgé des siècles passés auxquels il avait consacré sa thèse en architecture. Les plans prévus des appartements berlinois situés sous les toits, appartements choisis pour leur beauté ou leur originalité, semblent ignorer, eux aussi, la présence humaine. Images esthétisantes qui s'éloignent de la quotidienneté autant que celles de monuments ou de vues pittoresques des villes. S'agit-il d'une inconséquence dans ce projet qui se veut pourtant une alternative aux images que les actualités cinématographiques reproduisent inlassablement?

Sans nier le plaisir que pouvait avoir Kracauer à contempler ces formes, il faut tenir compte de la place qu'occupe ce court métrage dans la série proposée ${ }^{22}$. On s'aperçoit alors que, dès le court métrage suivant, l'attention se tourne du haut des beaux lieux déserts vers le bas, vers les lieux habités par les Berlinois, pour montrer les conditions d'habitation, ou plutôt leur changement dans la conjoncture du début des années 1930. De ces lieux habités, on passe ensuite aux règles qui régissent la vie quotidienne et les institutions administratives auxquelles sont confrontés les citadins. Enfin, le dernier court métrage de la série se penche sur le principal moyen d'information de l'époque, le journal.

2. «Le déménagement»- d'un grand vers un plus petit appartement, symptôme caractéristique des temps, est le deuxième sujet proposé. Quand il était encore à l'école et apprenait la destruction complète de grandes villes qui, d'un coup, étaient rayées de la carte, Kracauer rapporte, dans un texte écrit quelque temps auparavant et dont le sujet était également le déménagement, qu'il ne pouvait pas imaginer la fragilité des métropoles européennes, le caractère éphémère de Paris, Londres ou Berlin ${ }^{23}$. Pourtant, Berlin est menacé, non pas par une catastrophe subite, mais par un processus de décomposition plus ou moins long et

21. «Sous les toits de Paris», Kracauer, 2008, p. 113.

22. Kracauer travaille avec autant de minutie et de finesse ses textes que leurs articulations, en fait leur montage. Que l'on pense aux Employés ou à la composition de ses anthologies publiées après la guerre, L'ornement de la masse et Rues de Berlin et d'ailleurs. Voir Siegfried Kracauer, L'ornement de la masse. Essais sur la modernité weimarienne, trad. Sabine Cornille, édité par Olivier Agard et Philippe Despoix, Paris, La Découverte, 2008 et Siegfried Kracauer, Rues de Berlin et d'ailleurs, trad. Jean-François Boutout, Paris, Le Promeneur, 1995,

23. «3=6» (18.7.1931), dans Siegfried Kracauer, Berliner Nebeneinander. Ausgewählte Feuilletons 1930-33, édité par Andreas Volk, Zurich, Édition Epoca, 1996, p. 57-59. 
lent qui mine la ville de l'intérieur: «Des quartiers entiers languissent comme si la mort les avait marqués et, si la maladie n'est pas enrayée, se transforment plus ou moins rapidement en lotissements pour fantômes. Des champs de ruines entre des rues asphaltées - l'histoire atteint par des moyens toujours nouveaux et insoupçonnés ses vieux effets ${ }^{24}$.» Le mal a des racines socio économiques et le premier quartier touché est le vieux Berlin de l'Ouest. La plupart de ses villas cossues et de ses maisons patriciennes sont désormais vides. "Fatiguées, elles attendent avec nostalgie dans les jardins d'été comme des vieilles créatures, qui, mêlées aux arbres, continuent seulement à accueillir en elles des souvenirs ${ }^{25}$.» La description d'autres quartiers, moins nobles de la ville, conserve le même ton mélancolique, alors que la temporalité qui caractérise ce texte n'évoque en rien le rythme rapide, parfois effréné, qu’on trouve souvent associé à Berlin dans les portraits de villes et les miniatures urbaines de Kracauer.

Le ton change et l'ironie prend le pas sur la mélancolie dans la dernière partie du texte décrivant les projets que propriétaires, constructeurs et architectes développent pour sauver ce qui peut l'être encore. Une exposition des «Esquisses de division des grands et anciens appartements» a même eu lieu. On pouvait y voir comment d'un étage avec trois appartements on en fait un de six: « $3=6$ ». Lopération arithmétique était censée permettre de conserver les vieux immeubles et mettre sur le marché des appartements à des prix abordables pour le peuple. Les remèdes employés pour sauver les «maisons malades» ne sont cependant que partiellement ou plutôt superficiellement efficaces. Contrairement aux villes portuaires - Marseille en donne l'exemple -, les diverses couches de la population ne s'entremêlent pas à Berlin pour redonner vie à ces constructions. «Il semble alors que le dépérissement de ces quartiers est scellé26 », conclut-il.

Peu de temps après, dans un autre texte sur la ville de Berlin et les bouleversements qu'entraîne la crise économique, Kracauer, selon un procédé déjà utilisé et proprement cinématographique, rapproche, plus radicalement que ne le font les adjectifs et images utilisés dans « $3=6$ ", êtres animés et inanimés en décrivant le déplacement des meubles d'un endroit à l'autre. Une certaine empathie est inhérente à ce rapprochement, alors que l'ironie n'est pas absente de la description de leur triste sort, quand, trimballés à travers la ville, ils finissent par échouer dans un appartement bien plus petit que celui qu'ils avaient quitté. Leur destin est solidaire de celui de leurs propriétaires: «Je crains qu'ils ne doivent dorénavant

24. Ibid., p. 57.

25. Ibid., p. 58 .

26. Ibid., p. 59 
compter les centimètres carrés aussi parcimonieusement que leurs propriétaires leurs sous et que le temps de la splendeur est pour eux à jamais révolu ${ }^{27}$. »

Le court métrage consacré au déménagement devait, dans tous les cas, être un film autonome, précise Kracauer. Aussi bien l'assimilation du monde des hommes au monde des objets que le ton des textes sur le déménagement en tant qu'expression du changement des conditions de vie dans Berlin en ce début des années 1930 imposent l'idée du burlesque. Et l'on pense irrésistiblement à Laurel et Hardy et à leur camion de déménagement, tandis que la touche de mélancolie du premier texte évoque plutôt la figure de Chaplin.

3. «Tout ce qui est interdit chez nous » - Comme le deuxième sujet proposé pour les courts métrages sonores, celui-ci est inspiré d'aspects de la réalité urbaine auxquels le flâneur-sociologue Kracauer avait déjà consacré des chroniques dans le feuilleton de la Frankfurter Zeitung. Des passages sur les réglementations et interdictions administratives, dans leur absurdité, émaillent Les employés. On les retrouve ailleurs, comme par exemple dans le très beau texte consacré aux bureaux de placement, où Kracauer commente toute une série d'injonctions, avertissements et interdictions qui jalonnent le parcours à travers l'espace des chômeurs et qui culmine avec le texte suivant d'un panneau de la cage d'escalier: «Il est interdit de séjourner sans raison dans les escaliers ${ }^{28}$." Mais ces films montreraient aussi - on l'imagine - toutes les règles qui régissent l'espace urbain : des simples gestes des agents de la circulation commandant aux passants et aux conducteurs, aux voix autoritaires d'origine incertaine qui retentissent dans l'espace des grandes stations de métro en criant, peu avant le départ de chaque rame: «En arrière ${ }^{29}$ ! Q Que la représentation cinématographique de telles scènes soit à la fois «drôle et amère » témoigne de la volonté de Kracauer d'éviter le pathétique ainsi que de sa prédilection pour les formes burlesques.

4. «Le Monde et la Presse »- Le quatrième et dernier sujet proposé est en fait une «confrontation de différents événements avec le récit qu'en donne la presse. Ce film pourrait constituer une critique révélatrice des informations habituelles de la presse. » La fin du premier chapitre de son étude sur les Employés, qui peut aussi être lue comme un portrait de Berlin, se terminait par une confrontation médiale analogue: «[La vie] n’est nullement contenue dans les séries d'observations plus ou moins aléatoires des reportages, on ne la trouvera que dans la mosaïque que constituent des observations particulières au fur et à

27. «Möbel auf Reisen» (3.10.1931), ibid., p. 61.

28. «À propos des Bureaux de placement» (17.6.1930), dans Kracauer, 1995, p. 87.

29. «Mots de la rue» (7.7.1930), dans Kracauer, 2008, p. 100-101. 
mesure que l'on appréhende leur teneur. Le reportage donne une photographie de la vie; l'image de la vie, quant à elle, c'est une mosaïque de ce genre qui nous l'offrirait ${ }^{30}$.» L'image de la mosaïque renvoyait au propre texte de Kracauer, à sa visée et aux modalités de sa réalisation, à sa méthode et à sa forme, en même temps qu'elle l'inscrivait directement dans la controverse autour du reportage à la mode de la Neue Sachlichkeit. Par le truchement de cette image de la mosaïque, l'étude était rapprochée de certains films documentaires ou semi-documentaires sur la grande ville. Ici, la confrontation entre la presse écrite et le film renvoie, d'un côté, à une pratique autre du reportage cinématographique et apparaît ainsi comme une mise en abîme de la proposition de Kracauer; de l'autre, elle semble ne pas viser seulement la presse populaire à sensation, mais la presse dans son ensemble. Dans ce cas, elle pourrait se lire comme une référence allusive à la situation de la Frankfurter Zeitung, à la perte de son indépendance, et renvoyer également à la propre situation de Kracauer ${ }^{31}$.

\section{LA VIE CITADINE}

Le deuxième projet de film, conçu par Kracauer dans les premiers temps de son exil parisien, prend pour thème la promenade dominicale et s'intitule simplement «Dimanche $[\text { Sonntag }]^{32}$ ». Le titre de ce moyen métrage le situe dans la proximité de nombreux textes de Kracauer, qui traitent des loisirs des classes moyennes, de la mode du week-end, ou tout simplement du dimanche selon différentes perspectives: la ville vidée après la ruée vers la campagne le diman-

30. Kracauer, Les employés, 2004, p. 30.

31. Le problème de l'indépendance des journaux le préoccupe depuis ses débuts dans le journalisme. Voir par exemple l'article, écrit un an à peine après son entrée à la rédaction du journal, qui a pour thème justement l'indépendance des journaux allemands et les pressions qui, à l'époque, pouvaient s'exercer: «Die Zeitung» (16.9.1922), dans Siegfried Kracauer, Frankfurter Turmhäuser. Ausgewählte Feuilletons 1906-1930, Andreas Volk (dir.), Zurich, Édition Epoca, 1997, p. 275-277.

32. Le manuscrit, dactylographié, porte la première adresse à Paris de Kracauer, Hôtel Madison, 134 Bd. St-Germain, Paris $\left(6^{\circ}\right)$, où il a habité d'avril 1933 à octobre 1936. Il est daté de «Anfang Mai». Soit il a été écrit en mai 1933, soit il s'agit d'une version ultérieure, retravaillée d'un manuscrit envoyé en avril 1934 à Leo Lania à Londres, en même temps que quelques articles parus encore dans la FZ, avec l'indication suivante: «il s'agit d'une esquisse pour un film d'une longueur de 1000 mètres ». Dans la même lettre, Kracauer fait allusion à l'intérêt porté au projet par Jean Renoir. Dans les archives de Kracauer, il existe une version française du manuscrit, également datée «Début mai». Il s'agit d'une traduction assez fidèle mais qui, parfois, résume légèrement le texte allemand. 
che matin, ou les promenades du dimanche matin des habitants des «beaux quartiers », etc. ${ }^{33}$ Sur le plan thématique, le synopsis, assez détaillé, s'inscrit donc dans l'ensemble de sa production des années de Weimar. D'autre part, son titre évoque Les hommes le dimanche et sa structure suit effectivement le modèle des Querschnittfilme, son argument consistant à suivre la promenade dominicale d'une famille de petits-bourgeois ${ }^{34}$.

La partie principale du film, la promenade, est encadrée par un prologue et un épilogue qui se déroulent dans l'appartement familial. L'exposition décrivant l'intérieur montre les personnages, le père, la mère et la grand-mère, et les situe socialement. Alors que le petit enfant joue, le père s'impatiente et lui demande de saluer sa grand-mère. L'enfant grimpe alors sur les genoux de celle-ci et l'embrasse. À la parole du père, l'enfant répond par une action. Le passage de l'expression langagière au mouvement corporel et muet coïncide avec un changement radical de perspective que Kracauer désigne par le mot de «métamorphose». Elle consiste à épouser la perspective de l'enfant, perspective qui sera celle du film jusqu'au moment du retour à la maison, lors de l'épilogue.

Ainsi que le mot de «métamorphose » le signale, il ne s'agit pas simplement de montrer les choses à partir d'une caméra filmant depuis la position occupée dans l'espace par l'enfant, ou depuis une position proche. Il s'agit, plutôt, d'imaginer des équivalents de sa perception du monde, ce qui implique d'abord un changement d'échelle brutal, un bouleversement des relations entre grand et petit. Avec cet agrandissement, toute une série de transformations s'opère: fragmentation, ralenti ou accéléré des mouvements, changement de l'aspect des surfaces, de leur texture: elles peuvent paraître plus brillantes, plus

33. Voir «Sonntagsausflug» (5.5.1930), dans Kracauer, 1996, p. 43-45; «Sonntagmorgen » (20.1.1927), dans Kracauer, 1997, p. 64-66; en français, voir par exemple: «Moyenne montagne » (1926), dans Kracauer, 1995, p. 133-135. À tous ces textes, il faudrait ajouter au moins quelques passages des Employés (1929).

34. Kracauer a sévèrement critiqué à sa sortie Berlin, die Sinfonie der Großstadt (Berlin, symphonie d'une grande ville, 1927) de Ruttmann, alors qu'il a fait l'éloge du film de Vertov. Son attitude face aux Querschnittfilme est plus différenciée qu'on a pu le dire. Difficile à résumer, elle vise autant un formalisme qui subsume les matériaux dans des abstractions ornementales qu'une objectivité illusoire et son instrumentalisation idéologique. Sur ce sujet, voir Philippe Despoix, «Feuilleton et cinéma. Siegfried Kracauer, une esthétique du médium ", dans Éthiques du désenchantement. Essais sur la modernité allemande au début du siècle, Paris, L'Harmattan, 1995, en particulier p. 193-197 et Nia Perivolaropoulou, "La ville cinématographique ", Théorème, n 10, 2007, en particulier p. 18-22. 
lisses ou plus rugueuses; les sons changent également, la tonalité d'une voix est soudain plus importante que le sens des mots indistincts qu'elle profère; les mouvements s'amplifient, les visages se décomposent en leurs parties. Les apparences familières doivent être à tel point transformées qu'elles deviennent étranges, méconnaissables. Le monde perçu par l'enfant doit constituer une énigme visuelle et sonore pour les adultes, les spectateurs.

Depuis le moment du départ de la maison, la succession des séquences du film suit l'itinéraire de la famille tout au long de l'après-midi, dans l'autobus, le parc, puis au café en plein air et peu avant leur retour, au carrousel. L'enfant entre alors lui aussi dans un monde fantastique: «De beaux chevaux sellés l'attendent en piaffant. Porté par la musique fascinante de l'orgue de barbarie, il chevauche et plein du désir d'aventure il oublie tout ce qui l'entoure. Il galope à travers les paysages de ses livres d'images coloriés ${ }^{35}$.» C'est avec surprise qu'il retrouve ses parents au moment où le carrousel s'arrête. Mais le film continue à présenter le monde selon la perception de l'enfant. Ce n'est qu'après le retour à la maison, alors que l'enfant est en train de s'endormir et que le père commence à raconter leur promenade à la grand-mère, que la perspective normale se rétablit.

Subordonnée au récit du père, cette perspective est illustrée par une suite de plans qui constituent en même temps les solutions des énigmes visuelles de la partie principale du film. Lunivers retrouve ses dimensions habituelles et son aspect quotidien, normal. Les choses apparaissent dans leurs fonctions, les hommes et les femmes dans leurs rôles, tout ce qui, au regard de l'enfant était merveilleux, grandiose ou dangereux, redevient ordinaire. Les mots et les images coïncident. Le carrousel avait été pour l'enfant le moyen d'accéder au monde enchanté de ses livres d'images; le film pourrait devenir pour les spectateurs le moyen d'accéder au monde merveilleux d'un regard enfantin. Monde merveilleux et estrangé. Ce rapprochement du carrousel et du cinéma, comme moyens d'étonnement, d'éloignement de la perception ordinaire, d'enchantement, Kracauer l'avait déjà suggéré dans un texte de 1927 sur Paris:

D'innombrables carrousels vous emportent dans toutes les directions célestes $[\ldots]$ aucun avion n'avale les distances comme ils le font, aucun paysage de cinéma n'est aussi sauvage que la nature qui surgit des planches sur lesquelles elle est peinte. Les enfants sont expédiés dans l'autre monde, sur des locomotives et sur des boucs, ils ne voient ni père ni mère, et s'éloignent, abasourdis et craintifs ${ }^{36}$.

35. «Dimanche [Sonntag]. Ideen-Entwurf zu einem Kurzfilm», dans Kracauer, 2004 , vol. 6.3 , p. 525 .

36. «Carrousels» dans «Le peuple des rues de Paris», Kracauer, 1995, p. 143. 
«Dimanche» se détache de la production contemporaine de Kracauer en construisant clairement l'affinité entre perception cinématographique et perception enfantine. Il anticipe par là l'explicitation ultérieure de ce thème dans Theory of Film, alors que le remplacement des affinités entre le flâneur et le spectateur de cinéma par celles entre ce dernier et l'enfant annonce de facto un déplacement d'accent majeur dans sa réflexion théorique. En effet, dans les essais des années 1920 et 1930, les affinités du cinéma pour tout ce qui est transitoire et éphémère fondaient les liens entre la ville et le cinéma comme elles fondaient la perception dans la distraction du flâneur et celle du spectateur ${ }^{37}$. La rue, lieu de transit par excellence, acquérait «ce caractère à la fois poétique et philosophique qu'aura le passage chez Benjamin ${ }^{38}$ ». Dans Théorie du film, la rue semble avoir remplacé la ville. Elle fonctionne moins comme sa métonymie que comme un lieu défini, comme autrefois la ville, par des qualités propres qui correspondent aux affinités du médium cinématographique. C'est la rue qui sert à qualifier les espaces urbains cinématographiques; espaces clos, comme les bars et les gares, ou espaces ouverts, comme les routes ou les autoroutes. Mais elle s'étend au-delà de l'urbain, son flux s'identifiant avec le «flux de la vie», matériau de la caméra: «La rue au sens large du mot, écrit Kracauer, n’est pas seulement le théâtre des impressions fugitives et des rencontres de hasard, mais le lieu où le flux de la vie est amené à se manifester comme tel ${ }^{39}$.»

Espaces cinématographiques déterminés par les qualités intrinsèques du médium, la ville et la rue semblent déterritorialisées, délocalisées. Elles le sont effectivement, non pas au profit d'un espace virtuel et abstrait, mais à la faveur d'un changement de perspective théorique induit par la préoccupation devenue centrale dans les deux derniers ouvrages de Kracauer, Théorie du film et L'Histoire. Des avant-dernières choses ${ }^{40}$ : la réflexion sur la possibilité d'une expérience sensible qui présuppose le sauvetage de la réalité matérielle et sur

37. Sur cette évolution de la pensée de Kracauer relative aux rapports entre la ville, le cinéma et le sujet de la perception, voir Nia Perivolaropoulou, «Du flâneur au spectateur. Modernité, grande ville et cinéma chez Siegfried Kracauer», dans Stéphane Füzesséry et Philippe Simay, Le choc des métropoles. Simmel, Kracauer, Benjamin, Paris-Tel-Aviv, éditions de l'éclat, 2008, p. 125-148.

38. Philippe Despoix, «Siegfried Kracauer, chroniqueur de l'époque de Weimar», dans Kracauer, 2008, p. 11.

39. Kracauer, 2010, p. 123.

40. Siegried Kracauer, L'Histoire. Des avant-dernières choses, trad. Claude Orsoni, édité par Nia Perivolaropoulou et Philippe Despoix, présenté par Jacques Revel, Paris, Stock, 2006 . 
la corrélative perception esthétique de la réalité dans laquelle nous vivons. Les deux projets filmiques considérés ici corroborent les affinités profondes entre la grande ville et le cinéma telles qu'elles avaient été élaborées dans les autres textes de Kracauer des années 1920 et 1930. En même temps, ces textes destinés à la production cinématographique montrent que ce n'est pas uniquement la visibilité ou la temporalité qui sont en cause, mais la perception sensible dans son ensemble. Lespace urbain est de facto un espace acoustique, auquel appartient également la dimension langagière. Celle-ci représente, dans toutes ses dimensions (paroles, cris, chants...), un des éléments de l'espace social. À travers le cinéma, médium audiovisuel, Kracauer dévoile des aspects de cette dimension qui se situent en dehors de la communication ordinaire. Le jeu entre perceptions visuelle et acoustique ouvre alors vers une perception de la réalité estrangée. L’inconscient optique s'élargit vers l'idée d'un imperçu, que le cinéma permet de penser, alors même que la notion de visibilité, constitutive de la perception estrangée de la réalité, ne perd rien de son importance dans la réflexion kracauerienne, en particulier quant à la possibilité de la représentation de la Shoah.

Lus en résonance avec d'autres textes, les petits écrits de Kracauer pour le cinéma, bien que marginaux, laissent à la fois entrevoir l'entrelacement de ses différentes approches de la ville et in nuce les développements théoriques ultérieurs, en mettant à jour l'importance de l'expérience cinématographique et la centralité du médium film pour penser la réalité, y compris celle de la ville. Enfin, ils contredisent, eux aussi, toute périodisation rigide de l'œuvre fondée sur une opposition entre sa production de la période de la République de Weimar et celle de l'exil américain. 